\title{
General Solution of Linear Fractional Neutral Differential Difference Equations
}

\author{
Hai Zhang, ${ }^{1,2}$ Jinde Cao, ${ }^{1,3}$ and Wei Jiang ${ }^{4}$ \\ ${ }^{1}$ Department of Mathematics, Southeast University, Nanjing, Jiangsu 210096, China \\ ${ }^{2}$ Department of Mathematics, Anqing Normal University, Anqing, Anhui 246133, China \\ ${ }^{3}$ Department of Mathematics, King Abdulaziz University, Jeddah 21589, Saudi Arabia \\ ${ }^{4}$ School of Mathematical Sciences, Anhui University, Hefei, Anhui 230039, China
}

Correspondence should be addressed to Jinde Cao; jdcao@seu.edu.cn

Received 2 April 2013; Accepted 27 May 2013

Academic Editor: Pedro R. S. Antunes

Copyright (C) 2013 Hai Zhang et al. This is an open access article distributed under the Creative Commons Attribution License, which permits unrestricted use, distribution, and reproduction in any medium, provided the original work is properly cited.

\begin{abstract}
This paper is concerned with the general solution of linear fractional neutral differential difference equations. The exponential estimates of the solution and the variation of constant formula for linear fractional neutral differential difference equations are derived by using the Gronwall integral inequality and the Laplace transform method, respectively. The obtained results extend the corresponding ones of integer order linear ordinary differential equations and delay differential equations.
\end{abstract}

\section{Introduction}

Fractional differential equations have been proved to be an excellent tool in the modelling of many phenomena in various fields of engineering, physics, and economics. Many practical systems can be represented more accurately through fractional derivative formulation. For more details on fractional calculus theory, one can see the monographs of Miller and Ross [1], Podlubny [2], Magin [3], Diethelm [4], and Kilbas et al. [5]. Fractional differential equations without delay involving the Riemann-Liouville fractional derivative or the Caputo fractional derivative have been paid more and more attention in [6-20] and references therein. In [5], Kilbas et al. have discussed the explicit solutions of linear fractional ordinary differential equations based on the method of successive approximations. In [6,7], the theory of inequalities, local existence, extremal solutions, comparison results, and global existence of the solutions of fractional differential equations are established. In [8], Li et al. have developed an operator theory to study fractional Cauchy problems with the Riemann-Liouville fractional derivatives in infinite-dimensional Banach spaces. In [9], Bonilla et al. have considered the explicit solution for linear fractional ordinary differential equations employing the exponential matrix function and the fractional Green function. In [10], Odibat has derived the exact solution for the initial value problems of linear fractional ordinary differential systems by analytical approaches.

On the other hand, time delays are present inherently in many interconnected real systems due to transportation of energy and materials. For example, feedback control systems containing time delays and fractional processes and controllers lead to fractional delay systems. While delay differential systems with integer order have been thoroughly investigated during the past decades (see [21-23] and references therein), the research of fractional delay differential systems is still in the initial and developing stage [24-29].

Motivated and inspired by the mentioned works, in this paper, we investigate the representation of the general solution to linear fractional neutral differential difference system with the form

$$
\begin{gathered}
{ }^{c} D^{\alpha}[x(t)-C x(t-\tau)]=A x(t)+B x(t-\tau)+f(t), \quad t \geq 0, \\
x(t)=\varphi(t), \quad t \in[-\tau, 0],
\end{gathered}
$$

where ${ }^{c} D^{\alpha} x(t)$ denotes an $\alpha$ order Caputo fractional derivative of $x(t), 0<\alpha \leq 1, A, B$ are $n \times n$ constant matrices, 
$\tau$ is a constant with $\tau>0, f(t)$ is a $n$-dimensional continuous vector-valued function, $\varphi \in C^{1}\left([-\tau, 0], \mathbb{R}^{n}\right)$, and $C^{1}\left([-\tau, 0], \mathbb{R}^{n}\right)$ denotes space of continuously differentiable functions mapping the interval $[-\tau, 0]$ into $\mathbb{R}^{n}$.

As we all know, the Laplace transform method is an effective and convenient method for solving linear fractional differential equations. The exponential estimate of the solution is an indispensable tache, which guarantees the rationality of solving fractional differential equations by the Laplace transform method. In [11], Lin failed to take into consideration the exponential estimates of the solution, but the existence of the Laplace transform was taken for granted to solve fractional differential equations. The earlier studies concerning the Laplace transform of fractional differential equations can be found in $[1-5,11,16]$, which focused especially on the nondelayed case.

The purpose of this paper is to construct the representation of the general solution for system (1) by using the Laplace transform method. The exponential estimate for system (1) is presented by using the Gronwall integral inequality, which is basic to apply the Laplace transform. Moreover, the expression of the general solution for the homogeneous system and the variation of constant formula for system (1) are derived. The obtained results extend the corresponding ones of integer order linear ordinary differential equations and delay differential equations.

This paper is organized as follows. In the next section, we present some definitions and preliminary facts used in the paper. In Section 3, we derive the exponential estimates of the solutions for system (1), the expression of the general solution for the homogeneous system, and the variation of contant formula for system (1).

\section{Preliminaries}

In this section, we recall some definitions and preliminary facts which are used throughout this paper. For more details, one can see [1-5].

Definition 1. The Riemann-Liouville's fractional integral of order $\alpha>0$ for a function $f: R^{+} \rightarrow R^{n}$ is defined as

$$
D^{-\alpha} f(t)=\frac{1}{\Gamma(\alpha)} \int_{0}^{t}(t-s)^{\alpha-1} f(s) d s .
$$

Definition 2. The Caputo's fractional derivative of $\alpha(0 \leq m \leq$ $\alpha<m+1)$ for a function $f: R^{+} \rightarrow R^{n}$ is defined as

$$
{ }^{c} D^{\alpha} f(t)=\frac{1}{\Gamma(m-\alpha+1)} \int_{0}^{t}(t-s)^{m-\alpha} f^{(m+1)}(s) d s .
$$

Definition 3. The Laplace transform of a function $f(t)$ is defined as

$$
F(s)=£[f(t)]=\int_{0}^{+\infty} e^{-s t} f(t) d t, \quad s \in \mathbb{C},
$$

where $f(t)$ is $n$-dimensional vector-valued function.

From the above definitions, we know that if the integral (4) is convergent at the point $s_{0} \in \mathbb{C}$, then it converges absolutely for $s \in \mathbb{C}$ such that $\mathfrak{R}(s)>\mathfrak{R}\left(s_{0}\right)$. Moreover, for $\alpha \in(0,1]$, we have

$$
\begin{gathered}
£\left[{ }^{c} D^{\alpha} f(t)\right]=s^{\alpha} \mathfrak{E}[f(t)]-s^{\alpha-1} f(0), \\
£\left[{ }^{c} D^{-\alpha} f(t)\right]=s^{-\alpha} £[f(t)] .
\end{gathered}
$$

Lemma 4 (Gronwall integral inequality [21]). If $x(t)$ and $u(t)$ are real valued continuous functions on $[a, b]$, and $u(t)$ is a nondecreasing function on $[a, b]$. In addition, $g(t) \geq 0$ is integrable on $[a, b]$ with

$$
x(t) \leq u(t)+\int_{a}^{t} g(s) x(s) d s, \quad t \in[a, b],
$$

then

$$
x(t) \leq u(t) e^{\int_{a}^{t} g(s) d s}, \quad t \in[a, b] .
$$

\section{Main Results}

In this section, we derive the exponential estimation of the solution $x(t)$ for system (1) which depends on $\varphi$ and $f$ based on Gronwall integral inequality. These estimates are basic to the applications of the Laplace transform, and we obtain the representation of the general solution for linear homogeneous fractional neutral system and the variation of constant formula for linear nonhomogeneous fractional neutral system.

Theorem 5. Assume system (1) has a unique continuous solution $x(t)$, if $f(t)$ is continuous on $[0,+\infty)$, then there exist positive constants $M>0$ and $\sigma>0$, such that

$$
\|x(t)\| \leq\left[M\|\varphi\|+\frac{\sigma \tau^{\alpha} t^{\alpha}}{\Gamma(\alpha+1)\left(\ln M+N^{\alpha}\right)}\|F(t)\|\right] e^{\sigma t},
$$

where $\|F(t)\|=\sup _{s \in[0, t]}\|f(s)\|, \quad N=(\|A\|+\|B\|) / \Gamma(\alpha+1)$.

Proof. For $t \geq 0$, system (1) is equivalent to the following Volterra integral equation:

$$
\begin{aligned}
x(t)= & \varphi(0)-C \varphi(-\tau)+C x(t-\tau) \\
& +\frac{1}{\Gamma(\alpha)} \int_{0}^{t}(t-s)^{\alpha-1} f(s) d s \\
& +\frac{1}{\Gamma(\alpha)} \int_{0}^{t}(t-s)^{\alpha-1}[A x(s)+B x(s-\tau)] d s, \quad t \geq 0 .
\end{aligned}
$$

Let $\|\varphi\|=\sup _{\theta \in[-\tau, 0]}\|\varphi(\theta)\|$. Applying the appropriate property of the norm, it follows that

$$
\begin{aligned}
\|x(t)\| \leq & \|\varphi(0)\|+\|C\|\|\varphi(-\tau)\|+\|C\|\|x(t-\tau)\| \\
+\frac{1}{\Gamma(\alpha)} \int_{0}^{t}(t-s)^{\alpha-1}\|f(s)\| d s & +\frac{1}{\Gamma(\alpha)} \int_{0}^{t}(t-s)^{\alpha-1} \\
& \quad \times[\|A\|\|x(s)\|+\|B\|\|x(s-\tau)\|] d s .
\end{aligned}
$$


Let $\|y(t)\|=\sup _{\theta \in[-\tau, 0]}\|x(t+\theta)\|$. For $0 \leq t \leq \tau$, then

$$
\begin{aligned}
\|y(t)\| \leq & (1+2\|C\|)\|\varphi\| \\
& +\frac{1}{\Gamma(\alpha)} \int_{0}^{t}(t-s)^{\alpha-1} \\
& \times[\|A\|\|y(s)\|+\|B\|\|y(s)\|+\|f(s)\|] d s \\
= & (1+2\|C\|)\|\varphi\|+\frac{t^{\alpha}}{\Gamma(\alpha+1)}\|F(t)\| \\
& +\frac{\|A\|+\|B\|}{\Gamma(\alpha)} \int_{0}^{t}(t-s)^{\alpha-1}\|y(s)\| d s .
\end{aligned}
$$

An application of Lemma 4 yields that

$$
\begin{aligned}
\|y(t)\| \leq & {\left[(1+2\|C\|)\|\varphi\|+\frac{t^{\alpha}}{\Gamma(\alpha+1)}\|F(t)\|\right] } \\
& \times e^{((\|A\|+\|B\|) / \Gamma(\alpha+1)) t^{\alpha}}, \quad t \in[0, \tau] .
\end{aligned}
$$

Let $M=1+2\|C\|$ and $N=(\|A\|+\|B\|) / \Gamma(\alpha+1)$, then (12) can be estimated as

$$
\|y(t)\| \leq\left[M\|\varphi\|+\frac{t^{\alpha}}{\Gamma(\alpha+1)}\|F(t)\|\right] e^{N t^{\alpha}}, \quad t \in[0, \tau] .
$$

Moreover, the same argument implies the following estimation:

$$
\begin{array}{r}
\|y(t)\| \leq\left[M\left\|y\left(t_{0}\right)\right\|+\frac{\left(t-t_{0}\right)^{\alpha}}{\Gamma(\alpha+1)}\|F(t)\|\right] e^{N\left(t-t_{0}\right)^{\alpha}}, \\
t \in\left[t_{0}, t_{0}+\tau\right] .
\end{array}
$$

Next, we need to prove that

$$
\begin{array}{r}
\|y(t)\| \leq\left[M\|\varphi\|+\frac{\sigma \tau^{\alpha} t^{\alpha}}{\Gamma(\alpha+1)\left(\ln M+N^{\alpha}\right)}\|F(t)\|\right] e^{\sigma t^{\alpha}}, \\
t \in[0, k \tau],
\end{array}
$$

where $N=(\|A\|+\|B\|) / \Gamma(\alpha+1), \sigma>N+\tau^{-\alpha}$. According to (13), we know that (15) is true for $t \in[0, \tau]$. Assume that (15) is true for $t \in[0, k \tau], k \geq 1$. By this hypothesis, we need to prove that $(15)$ is true for $t \in[0,(k+1) \tau]$. For $t \in[\tau,(k+1) \tau]$, we denote $t_{1}=t-\tau \in[0, k \tau]$, then it yields that

$$
\begin{aligned}
& \|y(t)\| \leq\left[M\left\|y\left(t_{1}\right)\right\|+\frac{\left(t-t_{1}\right)^{\alpha}}{\Gamma(\alpha+1)}\|F(t)\|\right] e^{N\left(t-t_{1}\right)^{\alpha}} \\
& =\left[M\left\|y\left(t_{1}\right)\right\|+\frac{\tau^{\alpha}}{\Gamma(\alpha+1)}\|F(t)\|\right] e^{N \tau^{\alpha}} \\
& \leq\left\{M\left[M\|\varphi\|+\frac{\sigma \tau^{\alpha}(t-\tau)^{\alpha}\|F(t-\tau)\|}{\Gamma(\alpha+1)\left(\ln M+N^{\alpha}\right)}\right]\right. \\
& \left.\times e^{\sigma(t-\tau)^{\alpha}}+\frac{\tau^{\alpha}\|F(t)\|}{\Gamma(\alpha+1)}\right\} e^{N \tau^{\alpha}} \\
& \leq\left\{\left[M\|\varphi\|+\frac{\sigma \tau^{\alpha}(t-\tau)^{\alpha}\|F(t-\tau)\|}{\Gamma(\alpha+1)\left(\ln M+N^{\alpha}\right)}\right]\right. \\
& \left.\times e^{\sigma(t-\tau)^{\alpha}+N \tau^{\alpha}+\ln M}+\frac{\tau^{\alpha}\|F(t)\|}{\Gamma(\alpha+1)}\right\} e^{N \tau^{\alpha}} \\
& \leq\left\{\left[M\|\varphi\|+\frac{\sigma \tau^{\alpha}(t-\tau)^{\alpha}\|F(t)\|}{\Gamma(\alpha+1)\left(\ln M+N^{\alpha}\right)}\right]\right. \\
& \left.\times e^{\sigma(t-\tau)^{\alpha}+N \tau^{\alpha}+\ln M}+\frac{\tau^{\alpha}\|F(t)\|}{\Gamma(\alpha+1)}\right\} e^{N \tau^{\alpha}} \\
& \leq\left[M\|\varphi\|+\frac{\sigma \tau^{\alpha}(t-\tau)^{\alpha}+\tau^{\alpha}\left(\ln M+N^{\alpha}\right)}{\Gamma(\alpha+1)\left(\ln M+N^{\alpha}\right)}\|F(t)\|\right] e^{\sigma t^{\alpha}} \\
& \leq\left[M\|\varphi\|+\frac{\sigma \tau^{\alpha} t^{\alpha}}{\Gamma(\alpha+1)\left(\ln M+N^{\alpha}\right)}\|F(t)\|\right] e^{\sigma t} \text {. }
\end{aligned}
$$

Thus, the proof is completed.

Remark 6. From the proof of Theorem 5, we know that the exponential estimation of the solution $x(t)$ for system (1) depends on $\varphi$ and $f$, then we denote solution $x(t)$ for system (1) as $x(t, \varphi, f)$.

Remark 7. From Theorem 5 , if $f(t)$ is continuous on $[0,+\infty)$ and exponentially bounded, then $x(t)$ is exponentially bounded, which is basic to apply the Laplace transform.

Next, we consider the general solution of linear homogeneous equation of the form

$$
{ }^{c} D^{\alpha}[x(t)-C x(t-\tau)]=A x(t)+B x(t-\tau) .
$$

The fundamental solution of the homogeneous system (17) is defined as follows:

Let $X(t) \in \mathbb{R}^{n \times n}$, and satisfy

$$
\begin{gathered}
{ }^{c} D^{\alpha}[X(t)-C X(t-\tau)]=A X(t)+B X(t-\tau), \\
X(t)= \begin{cases}I, & t=0, \\
0, & t \in[-\tau, 0),\end{cases}
\end{gathered}
$$

then $X(t)$ is called the corresponding fundamental solution of system (17). 
Let

$$
H(s)=s^{\alpha} I-A-e^{-s \tau} B-s^{\alpha} e^{-s \tau} C
$$

and $\mathfrak{E}^{-1}$ denote inverse transform of the Laplace transform. Applying the Laplace transform to system (17), from (18), we have

$$
X(t)=\mathfrak{E}^{-1}\left[s^{\alpha-1} H^{-1}(s)\right] .
$$

In terms of the fundamental solution $X(t)$ of system (17), the general solution $x(t, \varphi, 0)$ for system (17) can be represented in the following theorem.

Theorem 8. If $X(t)$ is the fundamental solution of system (17), then the general solution $x(t, \varphi, 0)$ of system (17) can be represented in the following form:

$$
\begin{aligned}
x(t, \varphi, 0)= & X(t)[\varphi(0)-C \varphi(-\tau)] \\
& +B \int_{-\tau}^{0}\left[\left({ }^{c} D^{1-\alpha} X\right)(t-\tau-\theta)\right] \varphi(\theta) d \theta \\
& +B \int_{-\tau}^{0} \frac{(t-\tau-\theta)^{\alpha-1}}{\Gamma(\alpha)} \varphi(\theta) d \theta \\
& +C \int_{-\tau}^{0} \dot{X}(t-\tau-\theta) \varphi(\theta) d \theta \\
& -C \int_{-\tau}^{0}[d X(t-\tau-\theta)] \varphi(\theta) d \theta .
\end{aligned}
$$

Proof. Applying the Laplace transform to system (17), we have

$$
\begin{aligned}
& s^{\alpha} \mathfrak{E}[x(t)-C x(t-\tau)]-s^{\alpha-1} \varphi(0)+s^{\alpha-1} C \varphi(-\tau) \\
&=A £[x(t)]+B £[x(t-\tau)] .
\end{aligned}
$$

It follows from the properties of integral that

$$
\begin{aligned}
£[x(t-\tau)] & =\int_{0}^{+\infty} e^{-s t} x(t-\tau) d t \\
& =e^{-s \tau} \mathfrak{E}[x(t)]+e^{-s \tau} \int_{-\tau}^{0} e^{-s t} \varphi(t) d t .
\end{aligned}
$$

Taking into account (22) and (23), it yields that

$$
\begin{aligned}
H(s) £[x(t)]= & s^{\alpha-1} \varphi(0)-s^{\alpha-1} C \varphi(-\tau) \\
& +B e^{-s \tau} \int_{-\tau}^{0} e^{-s t} \varphi(t) \\
& +s^{\alpha} C e^{-s \tau} \int_{-\tau}^{0} e^{-s t} \varphi(t) .
\end{aligned}
$$

Note that $H(s)=s^{\alpha} I-A-e^{-s \tau} B-s^{\alpha} e^{-s \tau} C$ and $s^{\alpha-1} H^{-1}(s)=$ $\mathfrak{E}[X(t)]$, we have

$$
\begin{aligned}
£[x(t)]= & £[X(t)][\varphi(0)-C \varphi(-\tau)] \\
& +£[X(t)] B s^{1-\alpha} e^{-s \tau} \int_{-\tau}^{0} e^{-s t} \varphi(t) d t \\
& +£[X(t)] C s e^{-s \tau} \int_{-\tau}^{0} e^{-s t} \varphi(t) d t .
\end{aligned}
$$

In order to apply the convolution theorem to the terms of the right in (25), we define a function $\omega(\cdot)$ and extend the initial function $\varphi(\cdot)$ as follows:

$$
\begin{gathered}
\omega(t)= \begin{cases}0, & t \geq 0, \\
1, & t \in[-\tau, 0),\end{cases} \\
\widehat{\varphi}(t)= \begin{cases}\varphi(0), & t \geq 0, \\
\varphi(t), & t \in[-\tau, 0) .\end{cases}
\end{gathered}
$$

Therefor, we have

$$
\begin{aligned}
\mathfrak{E} & {[X(t)] B s^{1-\alpha} e^{-s \tau} \int_{-\tau}^{0} e^{-s t} \varphi(t) d t } \\
& =B s^{1-\alpha} \mathfrak{E}[X(t)] e^{-s \tau} \int_{-\tau}^{+\infty} e^{-s t} \widehat{\varphi}(t) \omega(t) d t \\
& =B s^{1-\alpha} \mathfrak{E}[X(t)] \int_{0}^{+\infty} e^{-s t} \widehat{\varphi}(t-\tau) \omega(t-\tau) d t \\
& =B s^{1-\alpha} \mathfrak{E}[X(t)] £[\widehat{\varphi}(t-\tau) \omega(t-\tau)] .
\end{aligned}
$$

Moreover, the same argument implies the following equality:

$$
\begin{aligned}
& £[X(t)] C s e^{-s \tau} \int_{-\tau}^{0} e^{-s t} \varphi(t) d t \\
& \quad=C s £[X(t)] £[\widehat{\varphi}(t-\tau) \omega(t-\tau)] .
\end{aligned}
$$

Since

$$
\begin{gathered}
\mathfrak{E}\left[\left({ }^{c} D^{1-\alpha} X\right)(t)\right]=s^{1-\alpha} \mathfrak{E}[X(t)]-s^{-\alpha} X(0), \\
X(0)=I, \quad s^{-\alpha} \mathfrak{E}[x(t)]=\mathfrak{E}\left[D^{-\alpha} x(t)\right],
\end{gathered}
$$

then

$$
\begin{aligned}
£[x(t)]= & £[X(t)][\varphi(0)-C \varphi(-\tau)] \\
& +B £\left[{ }^{c} D^{1-\alpha} X(t)\right] £[\widehat{\varphi}(t-\tau) \omega(t-\tau)] \\
& +B £\left\{D^{-\alpha}[\widehat{\varphi}(t-\tau) \omega(t-\tau)]\right\} \\
& +C £[\dot{X}(t)] £[\widehat{\varphi}(t-\tau) \omega(t-\tau)] \\
& +C £[\widehat{\varphi}(t-\tau) \omega(t-\tau)] .
\end{aligned}
$$


The convolution theorem and inverse theorem of the Laplace transform applied to (30) yields the form

$$
\begin{aligned}
& x(t)=X(t)[\varphi(0)-C \varphi(-\tau)]+B\left[\left({ }^{c} D^{1-\alpha} X\right)(t)\right] \\
& *[\widehat{\varphi}(t-\tau) \omega(t-\tau)] \\
& +B D^{-\alpha}[\widehat{\varphi}(t-\tau) \omega(t-\tau)] \\
& +C[\dot{X}(t)] *[\widehat{\varphi}(t-\tau) \omega(t-\tau)] \\
& +C \widehat{\varphi}(t-\tau) \omega(t-\tau) \\
& =X(t)[\varphi(0)-C \varphi(-\tau)] \\
& +B \int_{0}^{t}\left[\left({ }^{c} D^{1-\alpha} X\right)(t-s)\right] \widehat{\varphi}(s-\tau) \omega(s-\tau) d s \\
& +B \int_{0}^{t} \frac{(t-s)^{\alpha-1}}{\Gamma(\alpha)} \widehat{\varphi}(s-\tau) \omega(s-\tau) d s \\
& +C \int_{0}^{t} \dot{X}(t-s) \widehat{\varphi}(s-\tau) \omega(s-\tau) d s \\
& +C \hat{\varphi}(t-\tau) \omega(t-\tau) \\
& =X(t)[\varphi(0)-C \varphi(-\tau)] \\
& +B \int_{0}^{\tau}\left[\left({ }^{c} D^{1-\alpha} X\right)(t-s)\right] \varphi(s-\tau) d s \\
& +B \int_{0}^{\tau} \frac{(t-s)^{\alpha-1}}{\Gamma(\alpha)} \varphi(s-\tau) d s \\
& +C \int_{0}^{\tau} \dot{X}(t-s) \varphi(s-\tau) d s \\
& +C \varphi(t-\tau) \omega(t-\tau) \text {. }
\end{aligned}
$$

Let $s=\tau+\theta$, then it follows from (31) that

$$
\begin{aligned}
x(t)= & X(t)[\varphi(0)-C \varphi(-\tau)] \\
& +B \int_{-\tau}^{0}\left[\left({ }^{c} D^{1-\alpha} X\right)(t-\tau-\theta)\right] \varphi(\theta) d \theta \\
& +B \int_{-\tau}^{0} \frac{(t-\tau-\theta)^{\alpha-1}}{\Gamma(\alpha)} \varphi(\theta) d \theta \\
& +C \int_{-\tau}^{0} \dot{X}(t-\tau-\theta) \varphi(\theta) d \theta \\
& +C \varphi(t-\tau) \omega(t-\tau),
\end{aligned}
$$

where $\omega(\theta)=1$ for $\theta<0$ and $\omega(\theta)=0$ for $\theta \geq 0$. If $t \geq \tau$, the term involving $\omega$ is not present. If $t<\tau$, then this term is $C \varphi(t-\tau)$ which is precisely the value of the Stieltjes integral $-\int_{-\tau}^{t-\tau}[d X(t-\tau-\theta)] \varphi(\theta) d \theta$. Therefore, if we make use of the Stieltjes integral, the relation for $x(t)$ can be written as

$$
\begin{aligned}
x(t)= & X(t)[\varphi(0)-C \varphi(-\tau)] \\
& +B \int_{-\tau}^{0}\left[\left({ }^{c} D^{1-\alpha} X\right)(t-\tau-\theta)\right] \varphi(\theta) d \theta \\
& +B \int_{-\tau}^{0} \frac{(t-\tau-\theta)^{\alpha-1}}{\Gamma(\alpha)} \varphi(\theta) d \theta \\
& +C \int_{-\tau}^{0} \dot{X}(t-\tau-\theta) \varphi(\theta) d \theta \\
& -C \int_{-\tau}^{0}[d X(t-\tau-\theta)] \varphi(\theta) d \theta .
\end{aligned}
$$

The proof is thus completed.

Remark 9. For the particular case $\alpha=1, B=C=0$, then formula (21) becomes

$$
x(t)=e^{A t} x(0)
$$

which is consistent with the classical result of the first-order linear differential equation with constant coefficient.

Remark 10. For the particular case $\alpha=1, C=0$, from Definitions 1 and 2, we have

$$
x(t)=X(t) \varphi(0)+B \int_{-\tau}^{0} X(t-\tau-\theta) \varphi(\theta) d \theta,
$$

which is consistent with the general solution of the first-order linear homogeneous delay differential equation [21].

Based on the Laplace transform method, we derive the variation of constant formula for the nonhomogeneous system (1).

Theorem 11. If $f(t)$ is continuous on $[0,+\infty)$ and exponentially bounded, and $X(t)$ is the fundamental solution of system (17), then the general solution $x^{*}(t, \varphi, f)$ of system (1) can be represented in the following form:

$$
\begin{aligned}
x^{*}(t, \varphi, f)= & X(t)[\varphi(0)-C \varphi(-\tau)] \\
& +B \int_{-\tau}^{0}\left[\left({ }^{c} D^{1-\alpha} X\right)(t-\tau-\theta)\right] \varphi(\theta) d \theta \\
& +B \int_{-\tau}^{0} \frac{(t-\tau-\theta)^{\alpha-1}}{\Gamma(\alpha)} \varphi(\theta) d \theta \\
& +C \int_{-\tau}^{0} \dot{X}(t-\tau-\theta) \varphi(\theta) d \theta+D^{-\alpha} f(t) \\
& -C \int_{-\tau}^{0}[d X(t-\tau-\theta)] \varphi(\theta) d \theta \\
& +\int_{0}^{t}\left({ }^{c} D^{1-\alpha} X\right)(t-\theta) f(\theta) d \theta .
\end{aligned}
$$


Proof. Applying the Laplace transform to system (1), it yields that

$$
\begin{aligned}
s^{\alpha} £ & {\left[x^{*}(t)-C x^{*}(t-\tau)\right]-s^{\alpha-1}[\varphi(0)-C \varphi(-\tau)] } \\
& =A £\left[x^{*}(t)\right]+B £\left[x^{*}(t-\tau)\right]+£[f(t)] .
\end{aligned}
$$

Note that $H(s)=s^{\alpha} I-A-e^{-s \tau} B-s^{\alpha} e^{-s \tau} C$ and $s^{\alpha-1} H^{-1}(s)=$ $\mathfrak{E}[X(t)]$, then we have

$$
\begin{aligned}
£\left[x^{*}(t)\right]= & £[X(t)][\varphi(0)-C \varphi(-\tau)] \\
& +£[X(t)] B s^{1-\alpha} e^{-s \tau} \int_{-\tau}^{0} e^{-s t} \varphi(t) d t \\
& +£[X(t)] C s e^{-s \tau} \int_{-\tau}^{0} e^{-s t} \varphi(t) d t \\
& +s^{1-\alpha} £[X(t)] £[f(t)] .
\end{aligned}
$$

According to the proof of Theorem 8, we have

$$
\mathfrak{E}\left[x^{*}(t)\right]=\mathfrak{E}[x(t)]+s^{1-\alpha} \mathfrak{E}[X(t)] \mathfrak{E}[f(t)] .
$$

Since

$$
\begin{gathered}
\mathfrak{E}\left[\left({ }^{c} D^{1-\alpha} X\right)(t)\right]=s^{1-\alpha} \mathfrak{E}[X(t)]-s^{-\alpha} X(0), \\
X(0)=I, \quad s^{-\alpha} \mathfrak{E}[f(t)]=\mathfrak{E}\left[D^{-\alpha} f(t)\right],
\end{gathered}
$$

then (39) can be represented in the following manner:

$$
\begin{aligned}
£\left[x^{*}(t)\right]= & £[x(t)]+£\left[\left({ }^{c} D^{1-\alpha} X\right)(t)\right] £[f(t)] \\
& +£\left[D^{-\alpha} f(t)\right] .
\end{aligned}
$$

The convolution theorem and inverse theorem of Laplace transform applied to (41) yields the form

$$
\begin{aligned}
x^{*}(t, \varphi, f)=x & (t, \varphi, 0)+D^{-\alpha} f(t) \\
& +\int_{0}^{t}\left({ }^{c} D^{1-\alpha} X\right)(t-s) f(s) d s .
\end{aligned}
$$

From Theorem 8 , we know that the solution $x(t, \varphi, 0)$ can be represented as

$$
\begin{aligned}
x(t, \varphi, 0)= & X(t)[\varphi(0)-C \varphi(-\tau)] \\
& +B \int_{-\tau}^{0}\left[\left({ }^{c} D^{1-\alpha} X\right)(t-\tau-\theta)\right] \varphi(\theta) d \theta \\
& +B \int_{-\tau}^{0} \frac{(t-\tau-\theta)^{\alpha-1}}{\Gamma(\alpha)} \varphi(\theta) d \theta \\
& +C \int_{-\tau}^{0} \dot{X}(t-\tau-\theta) \varphi(\theta) d \theta \\
& -C \int_{-\tau}^{0}[d X(t-\tau-\theta)] \varphi(\theta) d \theta
\end{aligned}
$$

hence, the general solution $x^{*}(t, \varphi, f)$ of system (1) can be represented as

$$
\begin{aligned}
x^{*}(t, \varphi, f)= & X(t)[\varphi(0)-C \varphi(-\tau)] \\
& +B \int_{-\tau}^{0}\left[\left({ }^{c} D^{1-\alpha} X\right)(t-\tau-\theta)\right] \varphi(\theta) d \theta \\
& +B \int_{-\tau}^{0} \frac{(t-\tau-\theta)^{\alpha-1}}{\Gamma(\alpha)} \varphi(\theta) d \theta \\
& +C \int_{-\tau}^{0} \dot{X}(t-\tau-\theta) \varphi(\theta) d \theta+D^{-\alpha} f(t) \\
& -C \int_{-\tau}^{0}[d X(t-\tau-\theta)] \varphi(\theta) d \theta \\
& +\int_{0}^{t}\left({ }^{c} D^{1-\alpha} X\right)(t-\theta) f(\theta) d \theta .
\end{aligned}
$$

The proof is thus completed.

Remark 12. For the particular case $\alpha=1, C=0$, from Definitions 1 and 2, we have

$$
\begin{aligned}
x^{*}(t, \varphi, f)= & X(t) \varphi(0)+B \int_{-\tau}^{0} X(t-\tau-\theta) \varphi(\theta) d \theta \\
& +\int_{0}^{t} X(t-\theta) f(\theta) d \theta,
\end{aligned}
$$

which is consistent with the general solution of the first-order linear nonhomogeneous delay differential equation [21].

\section{Acknowledgments}

The authors are very grateful to the Editor, Professor Pedro R. S. Antunes, and the two anonymous reviewers for their helpful and valuable comments and suggestions, which significantly contributed to improving the quality of the paper. This work is jointly supported by the National Natural Science Foundation of China under Grant nos. 61272530, 11072059, and 11071001, the Doctoral Fund of Ministry of Education of China under Grant no. 20093401110001, the Natural Science Foundation of Jiangsu Province of China under Grant no. BK2012741, and the Key Programs of Educational Commission of Anhui Province of China under Grant nos. KJ2010ZD02 and KJ2011A197.

\section{References}

[1] K. S. Miller and B. Ross, An Introduction to the Fractional Calculus and Fractional Differential Equations, John Wiley \& Sons, New York, NY, USA, 1993.

[2] I. Podlubny, Fractional Differential Equations, vol. 198 of Mathematics in Science and Engineering, Technical University of Kosice, Kosice, Slovak Republic, 1999.

[3] R. L. Magin, Fractional Calculus in Bioengineering, Begell House, Redding, Conn, USA, 2006. 
[4] K. Diethelm, The Analysis of Fractional Differential Equations: An Application-Oriented Exposition Using Differential Operators of Caputo Type, Springer, Berlin, Germany, 2010.

[5] A. A. Kilbas, H. M. Srivastava, and J. J. Trujillo, Theory and Applications of Fractional Differential Equations, vol. 204 of North-Holland Mathematics Studies, Elsevier Science B.V., Amsterdam, The Netherlands, 2006.

[6] V. Lakshmikantham and A. S. Vatsala, "Basic theory of fractional differential equations," Nonlinear Analysis. Theory, Methods \& Applications, vol. 69, no. 8, pp. 2677-2682, 2008.

[7] J. Q. Deng and L. F. Ma, "Existence and uniqueness of solutions of initial value problems for nonlinear fractional differential equations," Applied Mathematics Letters, vol. 23, no. 6, pp. 676680,2010

[8] K. X. Li, J. G. Peng, and J. X. Jia, "Cauchy problems for fractional differential equations with Riemann-Liouville fractional derivatives," Journal of Functional Analysis, vol. 263, no. 2, pp. 476-510, 2012.

[9] B. Bonilla, M. Rivero, and J. J. Trujillo, "On systems of linear fractional differential equations with constant coefficients," Applied Mathematics and Computation, vol. 187, no. 1, pp. 6878, 2007.

[10] Z. M. Odibat, "Analytic study on linear systems of fractional differential equations," Computers \& Mathematics with Applications, vol. 59, no. 3, pp. 1171-1183, 2010.

[11] W. Lin, "Global existence theory and chaos control of fractional differential equations," Journal of Mathematical Analysis and Applications, vol. 332, no. 1, pp. 709-726, 2007.

[12] X. Zhang, L. Liu, B. Wiwatanapataphee, and Y. Wu, "Positive solutions of eigenvalue problems for a class of fractional differential equations with derivatives," Abstract and Applied Analysis, vol. 2012, Article ID 512127, 16 pages, 2012.

[13] K. Sayevand, A. Golbabai, and A. Yildirim, "Analysis of differential equations of fractional order," Applied Mathematical Modelling, vol. 36, no. 9, pp. 4356-4364, 2012.

[14] Y. Zhou and F. Jiao, "Nonlocal Cauchy problem for fractional evolution equations," Nonlinear Analysis. Real World Applications, vol. 11, no. 5, pp. 4465-4475, 2010.

[15] J. Wang and Y. Zhou, "Analysis of nonlinear fractional control systems in Banach spaces," Nonlinear Analysis. Theory, Methods \& Applications, vol. 74, no. 17, pp. 5929-5942, 2011.

[16] K. X. Li and J. G. Peng, "Laplace transform and fractional differential equations," Applied Mathematics Letters, vol. 24, no. 12, pp. 2019-2023, 2011.

[17] J. Wang and H. Xiang, "Upper and lower solutions method for a class of singular fractional boundary value problems with $p$ Laplacian operator," Abstract and Applied Analysis, vol. 2010, Article ID 971824, 12 pages, 2010.

[18] X. Zhang, L. Liu, Y. Wu, and Y. Lu, "The iterative solutions of nonlinear fractional differential equations," Applied Mathematics and Computation, vol. 219, no. 9, pp. 4680-4691, 2013.

[19] R. Sakthivel, P. Revathi, and S. M. Anthoni, "Existence of pseudo almost automorphic mild solutions to stochastic fractional differential equations," Nonlinear Analysis. Theory, Methods \& Applications, vol. 75, no. 7, pp. 3339-3347, 2012.

[20] W. Q. Wu and X. B. Zhou, "Eigenvalue of fractional differential equations with Laplacian operator," Discrete Dynamics in Nature and Society, vol. 2013, Article ID 137890, 8 pages, 2013.

[21] J. K. Hale and S. M. Verduyn Lunel, Introduction to FunctionalDifferential Equations, Springer, New York, NY, USA, 1993.
[22] R. Manzanilla, L. G. Mármol, and C. J. Vanegas, "On the controllability of a differential equation with delayed and advanced arguments," Abstract and Applied Analysis, vol. 2010, Article ID 307409, 16 pages, 2010.

[23] X. S. Yang and J. D. Cao, "Synchronization of discontinuous neural networks with delays via adaptive control," Discrete Dynamics in Nature and Society, vol. 2013, Article ID 147164, 9 pages, 2013.

[24] V. Lakshmikantham, "Theory of fractional functional differential equations," Nonlinear Analysis. Theory, Methods \& Applications, vol. 69, no. 10, pp. 3337-3343, 2008.

[25] X. Y. Zhang, "Some results of linear fractional order time-delay system," Applied Mathematics and Computation, vol. 197, no. 1, pp. 407-411, 2008.

[26] Z. X. Zhang and W. Jiang, "Some results of the degenerate fractional differential system with delay," Computers \& Mathematics with Applications, vol. 62, no. 3, pp. 1284-1291, 2011.

[27] Y. Zhou, F. Jiao, and J. Li, "Existence and uniqueness for $p$-type fractional neutral differential equations," Nonlinear Analysis. Theory, Methods \& Applications, vol. 71, no. 7-8, pp. 2724-2733, 2009.

[28] F. L. Chen and Y. Zhou, "Attractivity of fractional functional differential equations," Computers \& Mathematics with Applications, vol. 62, no. 3, pp. 1359-1369, 2011.

[29] E. Kaslik and S. Sivasundaram, "Analytical and numerical methods for the stability analysis of linear fractional delay differential equations," Journal of Computational and Applied Mathematics, vol. 236, no. 16, pp. 4027-4041, 2012. 


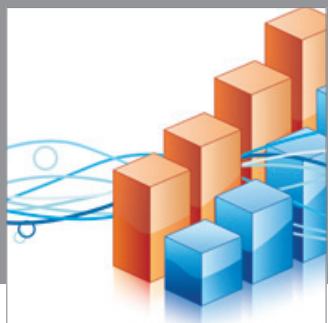

Advances in

Operations Research

mansans

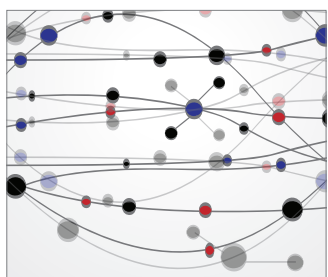

The Scientific World Journal
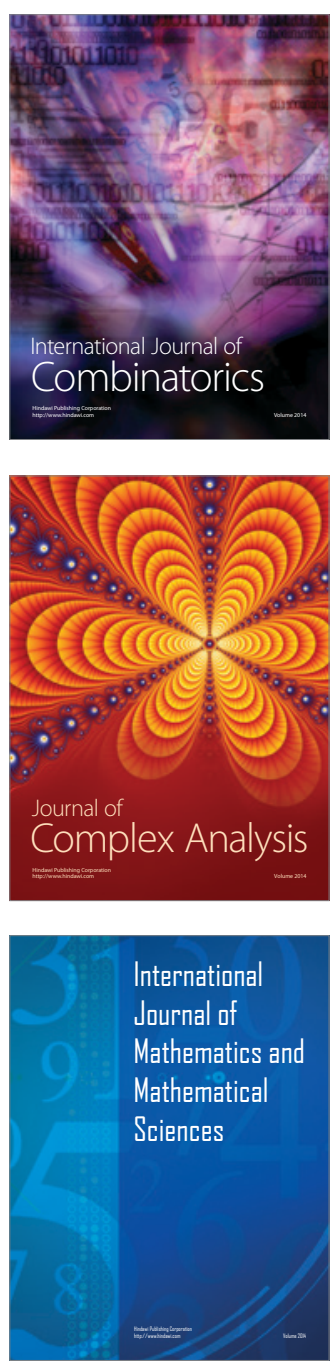
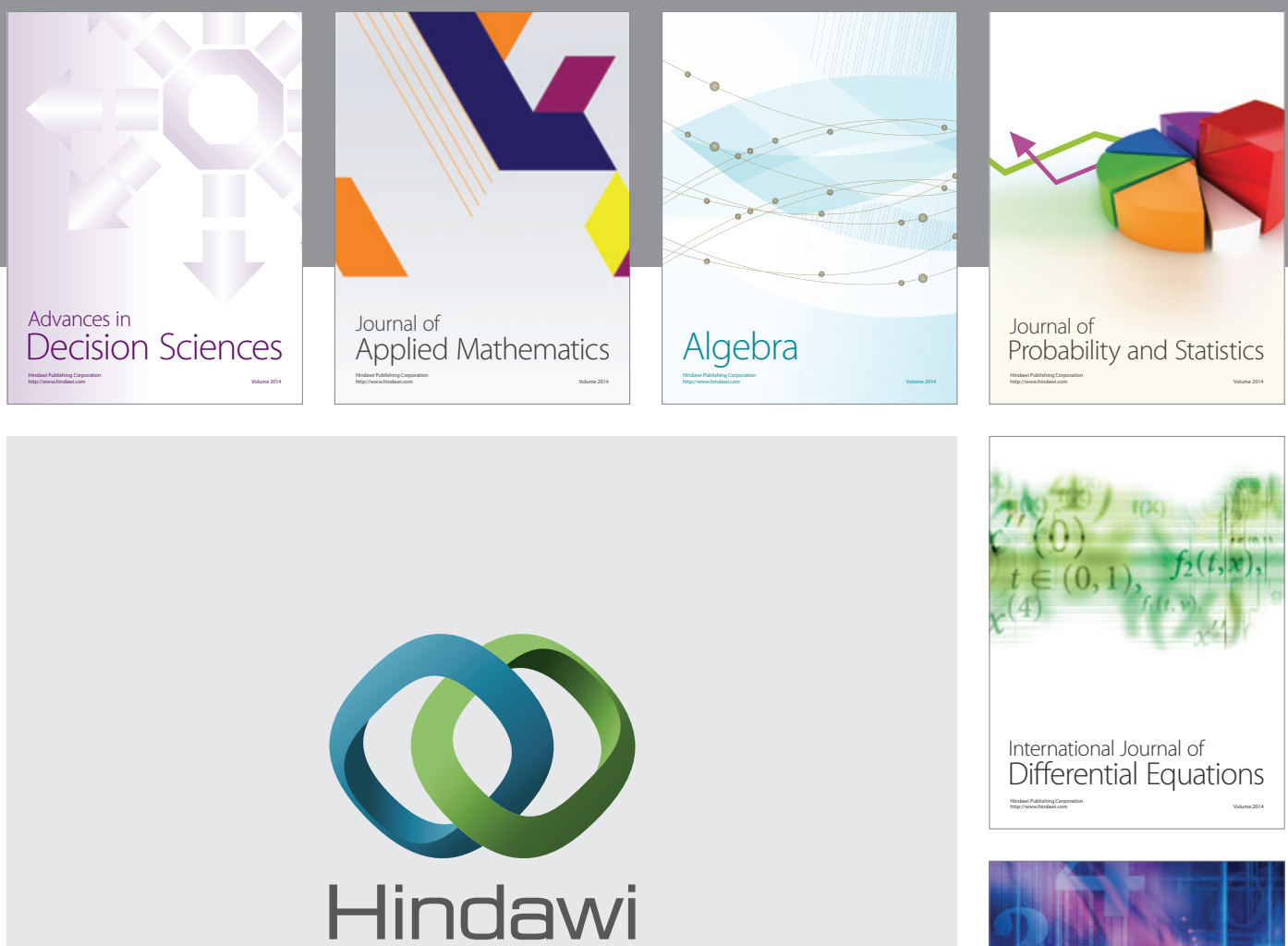

Submit your manuscripts at http://www.hindawi.com
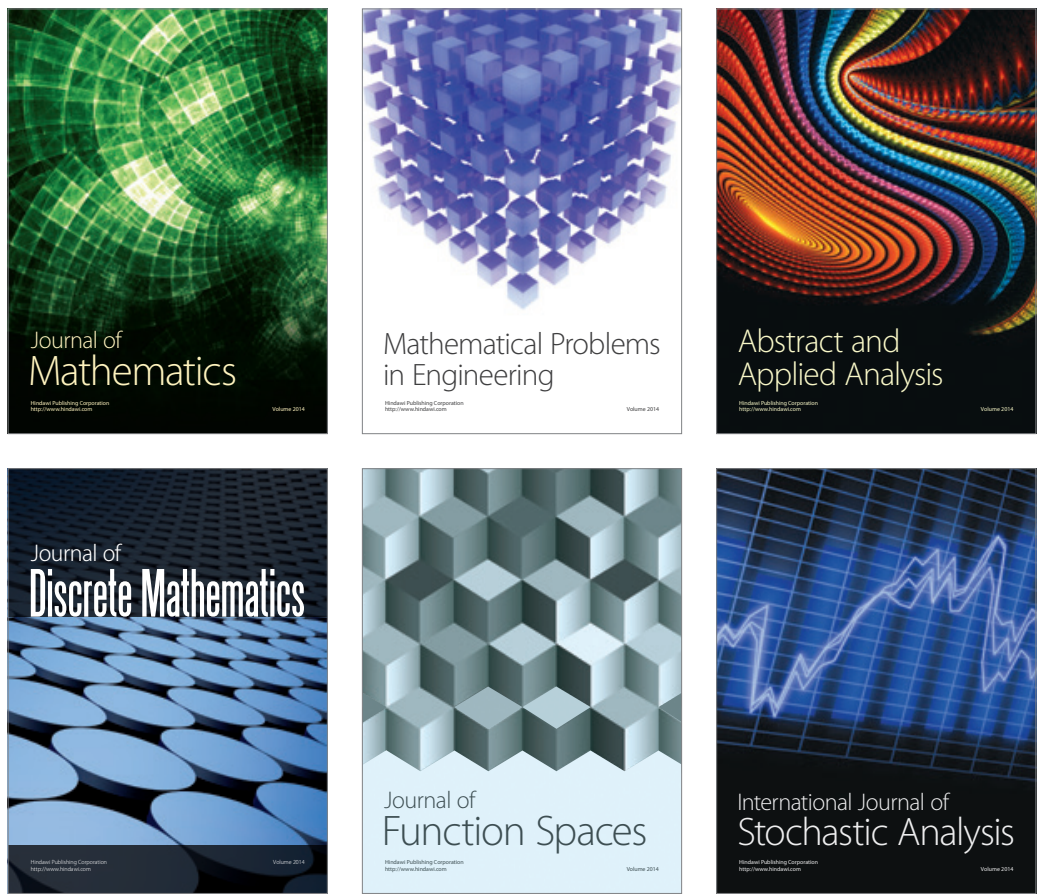

Journal of

Function Spaces

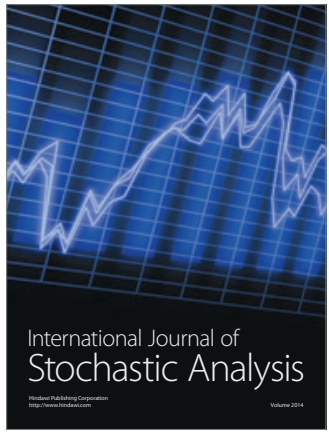

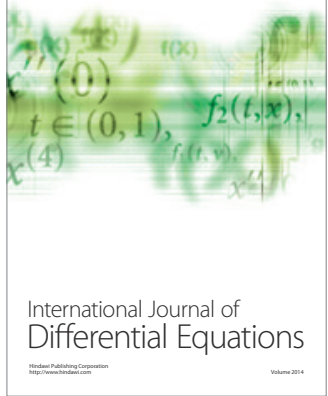
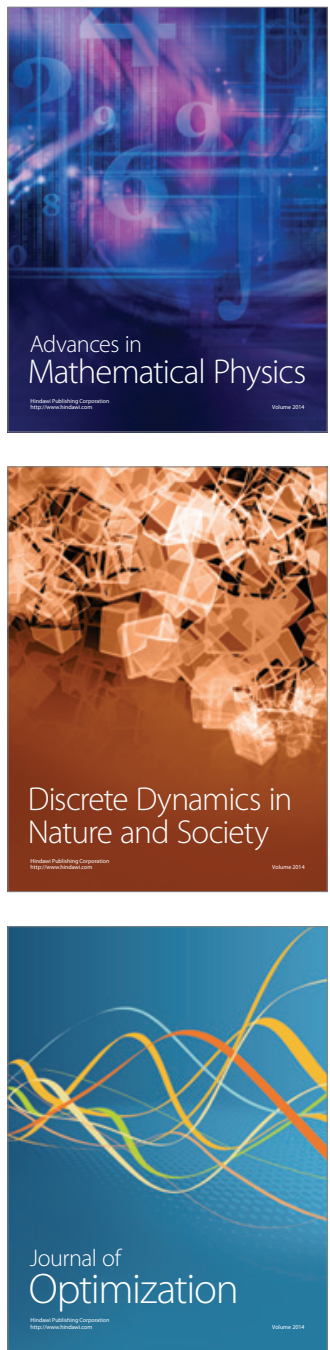